\title{
Corela
}

Cognition, représentation, langage

HS-15 | 2014

Complémentarité des approches qualitatives et quantitatives dans l'analyse des discours

\section{Concilier les données qualitatives et quantitatives : un défi pour les recherches en acquisition du langage}

Alessandra Del Ré et Rosângela Nogarini Hilário

\section{(2) OpenEdition}

\section{Journals}

Édition électronique

URL : http://journals.openedition.org/corela/3564

DOI : $10.4000 /$ corela.3564

ISSN : 1638-573X

Éditeur

Cercle linguistique du Centre et de l'Ouest - CerLICO

Référence électronique

Alessandra Del Ré et Rosângela Nogarini Hilário, « Concilier les données qualitatives et quantitatives : un défı pour les recherches en acquisition du langage », Corela [En ligne], HS-15 | 2014, mis en ligne le 15 octobre 2014, consulté le 30 avril 2019. URL : http://journals.openedition.org/corela/3564 ; DOI : 10.4000/corela.3564

Ce document a été généré automatiquement le 30 avril 2019

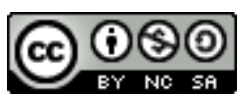

Corela - cognition, représentation, langage est mis à disposition selon les termes de la licence Creative Commons Attribution - Pas d'Utilisation Commerciale - Partage dans les Mêmes Conditions 4.0 International. 


\title{
Concilier les données qualitatives et quantitatives : un défi pour les recherches en acquisition du langage
}

\author{
Alessandra Del Ré et Rosângela Nogarini Hilário
}

\section{Introduction}

1 S'intéresser à l'acquisition du langage, c'est reconnaître, d'une part, la multiplicité des points de vue qui cernent ce domaine d'étude et, d'autre part, les frontières limitrophes qui la séparent de la linguistique, de la psychologie cognitive, de la psychanalyse, de la phono-audiologie, de la sociologie, de l'ethnologie et de l'éducation. En effet, malgré des fondements épistémologiques divers (théories générativiste, constructiviste ou behavioriste), des points communs existent dans le domaine de l'acquisition du langage : tout d'abord, leur intérêt pour le discours de l'enfant, c'est-à-dire pour un discours singulier qui présente ses propres caractéristiques, et ensuite la nécessité d'effectuer des recherches sur le terrain et de suivre les étapes de la collecte des données (sélection des sujets et choix du matériel), de manière à élaborer un corpus qui sera analysé et interprété.

2 La coexistence des approches qualitatives et quantitatives n'est pas une nouveauté dans le domaine de la psycholinguistique ou de l'acquisition du langage, mais si les études sur le langage reconnaissent l'importance de certains travaux de nature expérimentale, historiquement, elles ont toujours accordé une place privilégiée aux recherches prônant la singularité des données. Depuis quelques années, les programmes informatiques, qui assistent le travail des chercheurs avec des analyses automatiques et des calculs statistiques, ont gagné en notoriété et ont investi le champ des études linguistiques en général et, plus particulièrement, celui des recherches sur le langage de l'enfant. Actuellement, si tout semble aller de soi en ce qui concerne le choix de l'une ou l'autre approche, certains faits nous incitent à croire qu'en réalité, une certaine valeur semble 
attribuée au choix méthodologique ${ }^{1}$. Cette opposition reste voilée dans le sens où il n'existe pas - à notre connaissance - de travaux traitant frontalement de ces questions. Nous nous appuierons donc ici sur deux domaines connexes à notre discipline que sont la psychologie et l'analyse du discours.

Eu égard à l'exigence de rigueur scientifique, ainsi qu'à la comparaison constante qui est faite entre, d'un côté, les sciences humaines et, de l'autre, les sciences exactes, les sciences biologiques et les neurosciences dont les paramètres servent de modèle, il n'est pas surprenant de constater qu'il est de plus en plus exigé, de la part des études en acquisition du langage, des nombres, des quantités et des données statistiques pour valider les résultats obtenus ${ }^{2}$. Face à ces nouvelles exigences, il ne suffit pas d'ajouter des chiffres, des tableaux pour que les données qualitatives semblent plus vraisemblables. L'approche mixte, en acquisition du langage semble, pour l'instant, ne pas considérer la complémentarité comme le résultat des questions que le chercheur se pose, mais comme le moyen de faire accepter sa recherche auprès de la communauté scientifique.

La primauté revient aux données quantitatives et statistiques (Günther 2006; Valsiner 2005 ; 2006), c'est-à-dire à la recherche d'une réalité stable, objective, contrôlable et généralisable, alors que la singularité des données qualitatives présuppose une réalité dynamique, liée à une observation subjective, "naturaliste » et non contrôlable, dans laquelle le chercheur, étant très proche des données, oriente son regard vers le processus, vers la découverte exploratoire, descriptive et inductive ${ }^{3}$. Aujourd'hui, cette approche semble ne plus compter, alors qu'il s'agit pourtant de données authentiques et riches et qui sont maintes fois observées dans une étude de cas.

5 Au-delà de ces remarques, les recherches en acquisition du langage laissent au chercheur l'entière liberté de choisir sa méthodologie à partir de la théorie adoptée. Par conséquent, il n'existe pas de méthode unique, standardisée, mais plutôt une méthode qui s'adapte à chaque cas spécifique. Ainsi, il appartient à chaque chercheur, à partir de ses questions et de ses connaissances théoriques, de savoir quel type de relation il établira entre théorie et données empiriques, ou encore quel type de traitement il donnera à ce discours au moment de collecter les données et de les analyser (Del Ré, à par.).

6 Dès lors, on voit apparaître une relation inévitable entre le choix de l'approche (qualitative et/ou quantitative) et le phénomène à étudier, entre le concept théorique sur lequel se fonde l'étude, entre la méthodologie qui est suivie et la validité des réponses dans le domaine de l'acquisition du langage. Ainsi, plusieurs questions se posent sur la possibilité de généraliser (ou non) à partir de l'étude de cas et sur la « place » de l'analyse statistique (descriptive ou inférentielle) dans les recherches qui ont comme objet d'étude des phénomènes linguistiques et plus spécifiquement, la production et la compréhension orale des enfants au cours de leur période d'acquisition du langage.

7 L'enjeu consiste donc à concevoir la vraie complémentarité entre ces deux méthodologies : comment doit-on envisager une approche mixte? Faut-il compléter l'approche quantitative avec une approche qualitative (Mazière et al., 2010) ou faire le contraire? Comment ces deux approches peuvent-elles être combinées dans le cadre d'une même recherche en acquisition du langage ? Quelles sont les possibilités de généralisation qu'elles permettent?

8 Nous postulons d'une part, qu'il est possible d'élaborer une recherche dont les questions posées par le chercheur mènent à la complémentarité des approches et d'autre part, qu'il $\mathrm{y}$ a des cas où une approche mixte n'est pas nécessaire; dans ce cas, soit une seule 
approche sera adoptée, soit on pourra envisager d'introduire du quantitatif à l'intérieur du qualitatif (on y reviendra plus loin). A l'encontre d'une idée qui semble admise selon laquelle l'approche quantitative a une plus grande capacité de généralisation des données que les données qualitatives, nous défendrons donc l'idée que l'approche qualitative pourrait, tout comme l'approche quantitative, mener à des résultats tout aussi valides et permettre un certain type de généralisation.

Pour répondre à ces questions et vérifier notre hypothèse, nous présenterons cet article en trois parties: dans la première partie, nous exposerons notre cadre théorique qui privilégie une approche plutôt qualitative et dialogale. Nous essaierons ensuite de montrer comment introduire une approche quantitative dans l'approche qualitative et comment il est possible de concilier les deux approches et nous exposerons enfin l'approche choisie dans notre propre travail de recherche.

Dans la deuxième partie, nous discuterons des avantages et des limites de l'outil informatique dans l'analyse des données langagières des enfants, en prenant l'exemple du programme CLAN (CHAT) (MacWhinney \& Snow, 1990) et en illustrant notre propos par une étude de cas. Il s'agit d'un logiciel qui permet de réaliser des analyses qualitatives à partir de transcriptions qui sont toutes formatées de la même façon, grâce au format CHAT. Le logiciel CLAN, en faisant le lien entre une vidéo et sa transcription, rend possible une analyse fine de la relation entre les aspects verbaux et non-verbaux (gestes, situation etc.). CLAN permet également un traitement automatique des données issues de la transcription, qui peut donner lieu à une analyse de type quantitative. Par exemple, le programme MLT (« Mean Length of Turn») permet de calculer le nombre de mots ou de phrases par tour de parole, donnant ainsi la possibilité de comparer plusieurs enfants à différents âges. Dans ce cas, on peut dire qu'à partir d'une approche qualitative, on procède à une quantification qui ne correspond pas à la méthode quantitative telle qu'elle est utilisée dans les sciences "dures" (qualifiée d'approche hypothético-déductive).

Dans cet article, nous nous focaliserons sur l'articulation possible entre approche quantitative et approche qualitative en exposant une recherche réalisée sur les productions orales d'un enfant brésilien, enregistré à son domicile, en interaction naturelle avec son entourage pendant une année, entre l'âge de 2 et 3 ans. Nous nous intéresserons en particulier à l'acquisition des morphèmes du pluriel entre 1 an 11 mois $(1 ; 11)$ et 2 ans 7 mois $(2 ; 7)$.

Enfin, dans une troisième partie, nous reviendrons sur les apports et limites de la méthodologie que nous avons adoptée et nous ouvrirons la discussion sur une conciliation possible entre une approche expérimentale issue des sciences "dures » et une approche qualitative issue des sciences humaines afin de montrer que les deux sont nécessaires, et peuvent même être complémentaires, mais portent sur des perspectives différentes.

\section{Qualitatif/quantitatif et possibilités de généralisation en acquisition du langage}

\subsection{L'opposition qualitatif/quantitatif et la généralisation}

13 Il semble qu'il existe une croyance, basée sur le sens commun, qui veut que les données quantitatives soient " plus valables que les données qualitatives », car elles concernent de 
grandes populations, ce qui permet au chercheur de procéder à des généralisations. En fait, cette croyance se fonde sur la "Classifying Science» - adoptée par les sciences «dures»- selon laquelle plusieurs spécimens d'une même catégorie sont nécessaires pour parvenir à une connaissance fiable de cette catégorie, considérant que tous les membres d'une communauté ont les mêmes caractéristiques. Par conséquent, des échantillons de population sont nécessaires et un cas isolé ne permettrait pas de généraliser.

En adoptant ce présupposé, la psychologie de l'enfant a pu comparer, par exemple, des enfants d'âges différents, comme s'ils appartenaient à des groupes homogènes, et les a traités comme un objet social classable, dont le « classement en une catégorie » suffisait à expliquer le phénomène particulier qui justifiait à l'origine le dit «classement». De même, la psychologie de l'éducation et la pédagogie expérimentale ont commencé à traiter les enfants comme des spécimens qui formeraient des groupes similaires et, par conséquent, leur regard ne portaient plus sur des faits potentiellement remarquables. Ainsi, à travers l'expérience d'autres disciplines telles que la psychologie, il est possible de comprendre comment cette façon de traiter les données s'est imposée et a été adoptée par d'autres disciplines issues des sciences humaines, en particulier par une science voisine : la psycholinguistique. Cette pensée semble s'être développée jusqu'à nos jours (Valsiner, 2005 ; 2006) et s'est confondue avec le sens commun.

Mais, si nous considérons, comme le psychologue Gesell (1940: 7), que « deux enfants ne grandissent pas exactement de la même manière ", que "chaque enfant grandit selon un rythme et une manière qui lui est propre ", et si nous appliquons cette idée au domaine de l'acquisition, nous pouvons concevoir qu'en termes de processus d'acquisition du langage, deux enfants n'entrent pas nécessairement de la même manière dans la langue et/ou le langage. Ainsi, nous proposons de porter notre regard sur ce qui différencie les membres d'un même groupe. En ce sens, la généralisation pourrait se faire à partir des cas individuels de cette population. Si les populations sont des classes hétérogènes, il serait peut-être opportun d'étendre le concept d'échantillon et de l'envisager aussi en termes de différences. Dans ce cas, une théorie qui fonctionnerait aussi en fonction des différences pourrait également être valide.

Ce dont la science contemporaine a besoin, c'est de clarté, pour construire des méthodes appropriées selon des objectifs de recherche spécifiques, étant donné que chaque méthode présuppose des bases épistémologiques différentes (Sato et al. 2007: 91). Par conséquent, avant d'opter pour une approche quelconque - ou pour la complémentarité entre des approches différentes - le chercheur doit répondre à trois questions qui nous semblent fondamentales :

1) Une question ontologique : quelle est la nature du phénomène?

2) Une question téléologique : quelle est la finalité de la recherche?

3) Une question méthodologique : comment atteindre les objectifs ?

La question ontologique concerne la façon dont le phénomène étudié sera observé. Dans le cas où l'étude s'orienterait, à partir du contrôle des variables, vers quelque chose qui se répète, vers ce que les individus ont de typique et de commun, le chercheur pourrait parvenir, grâce à la manière dont il regarde le monde, à des lois générales applicables à partir de l'étude d'un groupe/échantillon (données quantitatives), dans une perspective nomothétique. D'un autre côté, dans le cas où l'objectif serait d'analyser ce qui est unique et singulier pour chaque phénomène, en cherchant à proposer de nouvelles théories, la 
constitution d'un corpus devrait également être singulière, avec une étude des cas individuels poussée en profondeur et en mettant l'accent sur l'importance du thème, des cas, et non sur la représentativité (perspective idiographique).

Pour ce dernier type, il convient de noter que le chercheur devra surmonter certaines difficultés : par exemple, le choix du cas qui sera pertinent à étudier - et la justification de ce choix -, l'inclusion et le découpage du contexte, le fait que les hypothèses et les échantillons peuvent être redéfinis et élaborés au cours de la recherche, et enfin la question de l'implication du chercheur. En dépit de ces difficultés et de la nature du regard qui est attribué à ce type d'étude, il est possible de procéder à des généralisations à partir de ce point de vue, contrairement à ce qu'on pourrait imaginer, comme nous le verrons par la suite.

En ce qui concerne la question téléologique, le chercheur doit avoir une idée claire de la finalité de son étude : si l'objectif est de comprendre la genèse d'un phénomène donné ou de caractériser les processus (ou les parties) qui composent ce phénomène, ou encore d'observer l'évolution du dit phénomène au cours du temps, etc. Ainsi, il s'agit pour le chercheur de définir les lignes à suivre pour atteindre ses objectifs.

Enfin, le chercheur se doit de réfléchir à la méthodologie la plus appropriée pour atteindre ses objectifs, et ce à partir du point de vue théorique qu'il a choisi. C'est à ce niveau que les détails des procédures adoptées pour la collecte des données sont mis au point. Il est bon de rappeler que les données n'existent pas a priori; le chercheur a une intuition éclairée sur la manière d'observer le phénomène - qui s'identifie avec la réflexion théorique apportée - et c'est ce processus qui fait surgir les données; le corpus, qui est un ensemble de données sur lequel s'effectuera le traitement analytique (personnes/groupes, lieu/événement, types de situation et nombre nécessaire des situations), constitue, en fait, un produit de la méthode (réflexions théoriques et méthodologiques).

\subsection{Variabilité des données et généralisation}

24 Ce sont les réponses du chercheur à ces trois questions fondamentales qui l'amèneront à réfléchir sur une généralisation possible de ses données et sur la façon de traiter la diversité et la variabilité. Il devra ainsi se demander ce qui sera le plus pertinent pour son étude :

a) la recherche de l'homogénéité, de ce qui est général dans la population (le découpage préserve la structure de l'univers de référence), c'est-à-dire une étude quantitative, ou

b) la recherche de l'hétérogénéité, afin de produire de nombreuses comparaisons, c'est-àdire une étude qualitative.

Quelle que soit la décision du chercheur, ces deux options aboutiront à différents types de généralisation.

En effet, le choix de l'homogénéité conduit à la généralisation statistique qui met l'accent sur la recherche des régularités. Ainsi, l'échantillon considéré sera celui des cas "égaux ", en préservant la «structure » de l'univers de référence, la similitude entre l'échantillon et la population, et en assurant sa représentativité.

Le choix de l'hétérogénéité conduit pour sa part vers la généralisation analytique qui se construit au fur et à mesure que de nouveaux cas sont étudiés et revus. Plus le phénomène est hétérogène, plus il est complexe. En ce sens, il s'agit d'examiner 
l'applicabilité du modèle à d'autres contextes : un modèle est tout d'abord créé pour l'étude d'un cas, puis des ajustements lui sont proposés pour le cas suivant. Moins il y a d'ajustements pour chaque nouveau et/ou différent cas et plus le modèle s'affirme. Il n'existe pas de nombre de cas minimum : un seul cas suffit pour étudier la singularité, car il permet de réfléchir. Mais, il en est tout autrement pour tester le modèle; plus le nombre de cas différents est élevé, mieux c'est.

Sur ce plan, la variabilité, c'est-à-dire les cas qui seraient exclus par le modèle nomothétique, ne pose pas de problème. Au contraire, selon le modèle systémique, qui part du présupposé que les composants d'un tout ne peuvent être isolés, il ne s'agit pas de généraliser des affirmations à partir de ce qui est égal, mais à partir de cas toujours plus distincts.

31 De même, toujours en ce qui concerne les recherches qualitatives, Günther (2006: 201), ainsi que Sato et al. (2007) et Valsiner (2007) proposent de généraliser les résultats (généralisation argumentative) à partir de cette approche. Le chercheur qui étaie ses recherches par des études de cas en lieu et place d'un échantillon représentatif, doit argumenter explicitement les généralisations possibles pour des circonstances spécifiques; il construit ses hypothèses et ses généralisations à partir d'éléments individuels (méthode inductive).

32 Face à ces questions concernant la généralisation et malgré les différences de point de vue qui peuvent amener le chercheur à opter pour l'une ou l'autre approche, il est possible dans certains cas, de penser à une triangulation méthodologique (Duarte, 2009) qui impliquerait la combinaison des méthodes quantitative et qualitative pour étudier un problème déterminé. Néanmoins, une telle complémentarité ne fonctionne que dans le cas où le phénomène en question est quantifiable et bien sûr, cela dépend de la nature du phénomène. Autrement dit, il ne s'agit pas ici de proposer la complémentarité pour valider un travail qualitatif au moyen de données quantitatives : ce sont les questions que le chercheur se pose, la nature de ces questions qui le conduiront éventuellement à une triangulation méthodologique, en cherchant des réponses dans le cadre des deux approches. Si l'on souhaite chercher des traces du processus d'acquisition du langage, durant un temps donné d'une ou plusieurs années, alors la méthode longitudinale et qualitative s'imposera. Si l'on s'intéresse au résultat à partir de modèles de similarité, alors la recherche expérimentale et quantitative sera mieux adaptée.

Comme l'a souligné Brunet (2007), bien qu'un corpus soit toujours artificiel étant donné que la nature n'en produit pas spontanément et que sa création est toujours guidée par la théorie et la méthodologie qui sous-tendent la recherche, les chercheurs en acquisition du langage se sont toujours efforcés de collecter des données recueillies en contexte aussi « naturel » que possible. Les chercheurs tentent de saisir en particulier le « parcours » de l'acquisition ou de considérer les facteurs discursifs et interactionnels afin de mieux comprendre comment s'effectue l'entrée de l'enfant dans le langage. Pour l'analyse de ce type de données, plusieurs facteurs sont à prendre en compte, contrairement à une étude dirigée où l'accent est mis sur le contrôle de certaines variables.

Quelque soit l'approche choisie, il sera possible d'arriver à un type de généralisation, comme proposé ci-dessus. Cependant, d'autres questions se posent rapport aux études quantitatives: sont-elles toujours généralisables? En fait, le niveau de généralisation dépendra $\mathrm{du}$ processus d'échantillonnage : dans un échantillonnage aléatoire (probabiliste), il est possible d'étendre les résultats et de considérer que l'échantillon peut supporter un haut niveau de généralisation. Mais, dans le cas d'un échantillon non- 
aléatoire (non probabiliste), le niveau de généralisation diminue considérablement. Il est bon de rappeler également que des erreurs sont tout de même possibles dans les études qui utilisent la statistique analytique, même si certaines techniques sont utilisées pour préserver l'étude. Ainsi, pour chaque étude, il est important que le chercheur pondère le niveau de généralisation qui est requis par sa discipline.

En ce qui concerne le travail que nous proposons en acquisition du langage et étant donné la théorie qui sert de base à nos propres études (Bakhtin, 1983 ; Vološinov, 1976 ; 1973), nous choisirons tout d'abord une approche qualitative, tout comme le proposait Bruner (1984; 2004). En effet, notre objectif est d'abord de décrire l'évolution du langage de l'enfant en nous demandant par exemple : quand est-ce qu'il commence à utiliser telle ou telle forme linguistique, dans quel contexte il le fait et quel est le rôle de l'interaction dans ce processus? Cette dernière question a aussi été discutée par Vygotski (1986). Nous exposerons ce type de questionnement à travers l'étude de l'acquisition de la marque morphologique du pluriel.

L'adoption de la théorie bakhtinienne et la proposition d'un rapport entre cette théorie et les études développées par Bruner et Vygotsky nous indiquent un chemin à suivre en ce qui concerne le choix de notre propre méthodologie. Pour ces auteurs, la production linguistique est intrinsèquement liée à son caractère dialogique et l'interaction (entre sujets et discours) joue un rôle très important. De ce fait, l'unité d'analyse la plus petite est l'énoncé, c'est-à-dire la production verbale insérée dans un contexte d'énonciation et dans le dialogue entre la mère et son enfant dans le domaine de l'acquisition.

\section{Une étude de cas : l'utilisation d'un outil informatique quantitatif dans une étude d'approche qualitative}

37 Dans le domaine de l'acquisition du langage et de la psycholinguistique, dès que les recherches sur le terrain sont terminées, on procède à la transcription des données. Pour ce faire, les outils informatiques sont de plus en plus utilisés, par exemple, le logiciel CLAN qui a été développé par MacWhinney et son équipe, et qui est disponible sur la plateforme CHILDES ${ }^{4}$ (MacWhinney, 1990). Outre le fait que le programme CLAN fournit un format pour la transcription des données (CHAT) - y compris la transcription phonétique -, il permet également d'associer le son et/ou la vidéo à la transcription. Ce programme met également à disposition de l'utilisateur différents outils pour une analyse " automatique » des énoncés, des mots et des morphèmes, et ce à partir d'une série de commandes qui peuvent être créées. Ces outils permettent au chercheur de comparer les productions linguistiques de différents enfants, de déterminer si elles sont plus ou moins communes et si ce n'est pas le cas d'attirer l'attention sur l'existence d'un éventuel retard de langage. Malgré les critiques faites au sujet du comptage, les outils du système CHILDES qui sont dédiés au lexique et à la morphosyntaxe (Parisse \& Le Normand, 1998 ; 2000a) favorisent une analyse fine des données, ce qui autorise un diagnostic à la fois précoce et précis et permet d'orienter les enfants vers des professionnels spécialisés.

En ce sens, ce genre d'outil informatique répond, en général, à la demande des chercheurs en sciences humaines qui souhaitent vérifier leurs données. Les résultats montrent que ces outils sont tout à fait fiables pour la réalisation de recherches quantitatives et suscitent un certain nombre de réflexions quant à leur rôle dans les études qualitatives. 
Plus précisément, en ce qui concerne les recherches menées par le groupe GEALin ${ }^{5}$ / NALingua $^{6}$, toutes essentiellement qualitatives, l'utilisation du logiciel CLAN pour la transcription de notre corpus longitudinal, permet non seulement de partager plus facilement avec les autres groupes de recherche, comme les groupes COLAJE et DIAREF en France $^{7}$, mais aussi de réduire considérablement le temps consacré à des analyses plus spécifiques, comme le calcul de la fréquence lexicale ou la description de l'utilisation de certains éléments lexicaux ou morphologiques (cf. tableaux ci-dessous).

Le regard du chercheur ne s'oriente pas sur ce qui pourrait être considéré comme une identification/rapprochement ou éloignement du discours de l'enfant par rapport à la production de l'adulte - ce qui pourrait être en soi une question pertinente - mais sur ce qui est particulier et qui peut être considéré comme une production localisée spécifiquement à un moment donné de l'acquisition. C'est le cas de la recherche que nous présenterons ensuite, à titre d'exemple, sur l'acquisition du pluriel en portugais du Brésil $(\mathrm{PB})^{8}$ chez un jeune enfant (Hilário, 2012).

41 En fait, ce regard, ancré dans un cadre théorique initial (travaux de Bakhtine et de son Cercle, ainsi que ceux de Bruner et de Vygotsky), prend en compte des réflexions sur la formation sociohistorique du langage, sur la notion d'un sujet qui construit son identité et sa subjectivité dans sa relation avec l'autre (exotopie) et sur les genres du discours.

Ce n'est donc pas ce qui est produit par l'enfant qu'il s'agit de découvrir, mais plutôt le processus mis en œuvre lorsque celui-ci s'efforce de marquer l'expression de la pluralité dans ses énoncés. À quel moment l'enfant commence à utiliser le morphème pluriel nominal ? Cet emploi se fait-il exclusivement d'une manière normative? Quels sont les contextes linguistiques/discursifs et les dimensions situationnelles qui amènent l'enfant à utiliser le pluriel ? Le discours de l'enfant s'identifie-t-il avec le discours de son interlocuteur adulte?

3 La question téléologique étant résolue, il s'agit maintenant de choisir la méthode. Une étude de nature générativiste a été proposée par Ferrari-Neto (2003) et Castro et FerrariNeto (2007) sur l'acquisition du pluriel en portugais du Brésil (désormais PB) et une comparaison avec le portugais du Portugal.

Dans ce cas, les chercheurs ont choisi la méthode expérimentale pour analyser la compréhension du morphème du pluriel - et non pas la production. Selon ces auteurs, les résultats semblent montrer que les enfants sont sensibles à l'expression grammaticale du nombre en PB, étant donné qu'ils se montrent capables de différencier les conditions grammaticales (marquage grammatical standard - déterminant et nom au pluriel - et non standard - déterminant au pluriel et nom au singulier) et non grammaticales (déterminant au singulier et nom au pluriel). L'analyse de la production, montre que la condition non grammaticale est davantage utilisée par l'enfant. Nous pouvons l'observer dans les deux exemples suivants, tirés de notre corpus :

Exemplo 1

Mãe (MOT) e criança (CHI, 2;2) brincam de enumerar as partes de um coelhinho de pelúcia

*MOT: que lin:da \# quantas orelhinhas ele tem?

*CHI: tem do:is \# t(r)rê:s \# quatro .

\%act: aponta para cada uma das orelhas do coelhinho enquanto conta

*MOT: nã:o \# conta uma +...

${ }^{*}$ CHI: uma...+

\%act: aponta para uma das orelhas do coelho

*MOT: duas +... 
${ }^{*}$ CHI: duas...+

\%act: aponta para a outra orelha do coelho

*MOT: duas orelhas .

${ }^{*} \mathrm{CHI}$ : dua(s) orelhas .

\%act: mostra dois dedos

${ }^{*}$ CHI: e a mão mãe ?

*MOT: quantas mãos?

${ }^{*}$ CHI: dois...+

\%act: aponta para uma das mãos do coelhinho

*MOT: +< uma +...

${ }^{*}$ CHI: uma...+

\%act: aponta para um das mãos do coelho

*MOT: du [//] duas .

\%com: CHI aponta para a outra mão do coelho

${ }^{*} \mathrm{CHI}$ : duas .

*MOT: <duas> [>] +/

${ }^{*}$ CHI: e...+

*CHI: e o pé ?

\%act: segura o coelhinho pelos pés

*MOT: um +...

${ }^{*}$ CHI: um...+

\%act: aponta para um dos pés do coelhinho

*MOT: dois .

${ }^{*}$ CHI: dois .

\%act: aponta para o outro pé do coelhinho

*MOT: dois [/] dois pés .

${ }^{*}$ CHI: doi(s) pés .

\%com: a criança suprime a fricativa da palavra « dois »

*MOT: e duas \# mãos .

*CHI: $e$ o olhos?

\%com: a criança suprime o morfema de plural do determinante

\%act: aponta para os olhos do coelhinho

*MOT: olhos [//] olhos \# um +...

*CHI: ó@i +...

\%act: aponta

$*_{\text {SIS: }}<$ dois $>[>]$.

*MOT: <dois $>[<]$.

*MOT: dois olhos .

Exemple 1 (traduction en français)

La mère (MOT) et l'enfant (CHI, 2;2) s'amusent à énumérer les différentes parties

d'un petit lapin en peluche.

*MOT: comme il est jo:li \# combien de petites oreilles a-t-il ?

*CHI: il a de:ux \# t(r)oi:s \# quatre .

\%act: il montre chaque oreille du lapin pendant qu'il compte

*MOT: no:n \# compte une +...

${ }^{*}$ CHI: une...+

\%act: il montre une des oreilles du lapin

*MOT: deux...+

${ }^{*}$ CHI: deux...+

\%act: il montre l'autre oreille du lapin

*MOT: deux oreilles .

${ }^{*} \mathrm{CHI}$ : $\operatorname{deu}(x)$ oreilles .

\%com: l'enfant supprime la fricative du mot « duas » (deux)

\%act: il montre deux doigts

*CHI: et la main maman?

*MOT: combien de mains? 
${ }^{*}$ CHI: deux $+\ldots$

\%act: il montre une des mains du petit lapin

*MOT: +< une +...

${ }^{*}$ CHI: une...+

\%act: il montre une des mains du lapin

*MOT: de [//] deux .

\%com: CHI montre l'autre main du lapin

${ }^{*}$ CHI: deux .

*MOT: $<$ deux $>[>]+/$

${ }^{*}$ CHI: et...+

${ }^{*} \mathrm{CHI}$ : et le pied?

\%act: il tient le lapin par les pieds

*MOT: un +...

${ }^{*}$ CHI: un...+

\%act: il montre un des pieds du lapin

*MOT: deux .

${ }^{*}$ CHI: deux .

\%act: il montre un autre pied du lapin

*MOT: deux [/] deux pieds .

${ }^{*} \mathrm{CHI}$ : deu(x) pieds .

\%com: l'enfant supprime la fricative du mot « dois » (deux)

*MOT: et deux \# mains.

${ }^{*} \mathrm{CHI}$ : et le yeux ?

\%com: l'enfant supprime le morphème du pluriel au déterminant

\%act: il montre les yeux du lapin

*MOT: yeux $[/ /]$ yeux \# un +...

*CHI: ó@i +...

\%act: il montre

${ }^{*}$ SIS: $<$ deux $>[>]$.

*MOT: $<$ deux $>[<]$.

*MOT: deux yeux.

Exemplo 2

Mãe (MOT) e criança (CHI, 2;3.13) estão na sala da casa, brincando de recortar e colar figuras.

${ }^{*} \mathrm{CHI}$ : agora vo(u) corta(r) a bolsas !

\%com: a criança suprime o morfema de plural do determinante

${ }^{*} \mathrm{CHI}$ : o(u)tra bolsa .

*MOT: eu acho que acabo(u) bolsa +...

*CHI: só tem ro(u)pa .

*MOT: tem bota ó@i .

*CHI: xxx .

${ }^{*}$ CHI: cadê a o(u)tra bolsa?

*MOT: (a)cabou as bolsas \# corta a bota preta +...

${ }^{*}$ CHI: preta .

Exemple 2 (traduction en français)

La mère (MOT) et l'enfant (CHI, 2;3.13) sont à la maison, ils s'amusent à découper et

à coller des figures.

${ }^{*} \mathrm{CHI}$ : maintenant je vais couper le sacs !

\%com: l'enfant supprime le morphème du pluriel au déterminant

${ }^{*} \mathrm{CHI}$ : autre sac .

*MOT:je pense que c'est fini le sac...+

*CHI: y'a que le vêtement.

*MOT: il y a la botte ó@i.

${ }^{*} \mathrm{CHI}$ : xxx .

${ }^{*} \mathrm{CHI}$ : où est l'autre sac? 
*MOT: c'est fini les sacs \# coupe la botte noire..+

*CHI: noire . pluralisés, avec un usage conventionnel ou non du morphème pluriel (cf. graphique 1, cidessous).

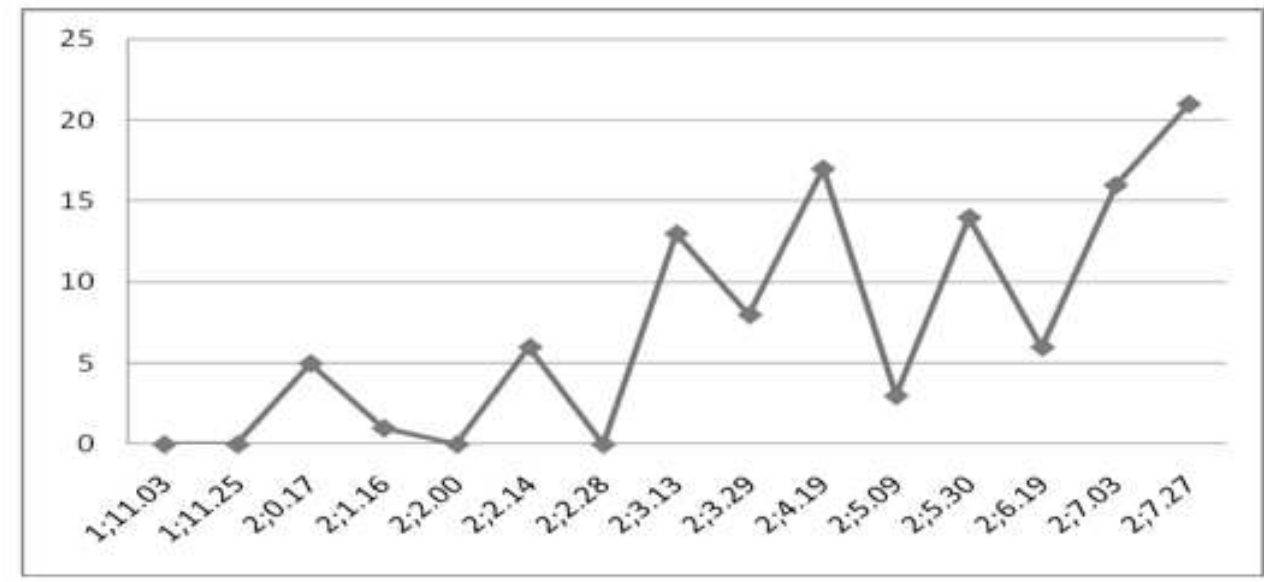

Graphique 1 : Nombre d'occurrences d'énoncés contenant la marque du pluriel chez A. entre l'âge de $1 ; 11.03$ et 2;7.27.

Nos objectifs nous ont conduites à choisir des données longitudinales et naturalistes, car selon notre approche théorique, l'observation doit porter sur la production de l'enfant dans des contextes réels d'énonciation, ainsi que sur la relation qui se tisse immanquablement entre son discours et celui de son interlocuteur adulte (en l'occurrence sa mère). Il s'agit donc tout d'abord d'une méthode qualitative. Cependant, le choix d'un corpus longitudinal et enregistré dans des situations quotidiennes ne nous a pas empêchés de montrer ce qui était régulier et récurrent dans le cas de la production du pluriel par l'enfant.

En ce qui concerne l'utilisation du logiciel CLAN, il nous a permis d'extraire et de compter ans le corpus, tous les énoncés produits par l'enfant qui comprennent des items lexicaux

Ainsi, l'utilisation systématique d'un accord pluriel dans le syntagme nominal a été observée, ce qui est atypique en PB (déterminant au singulier et nom au pluriel) par rapport à la production adulte.

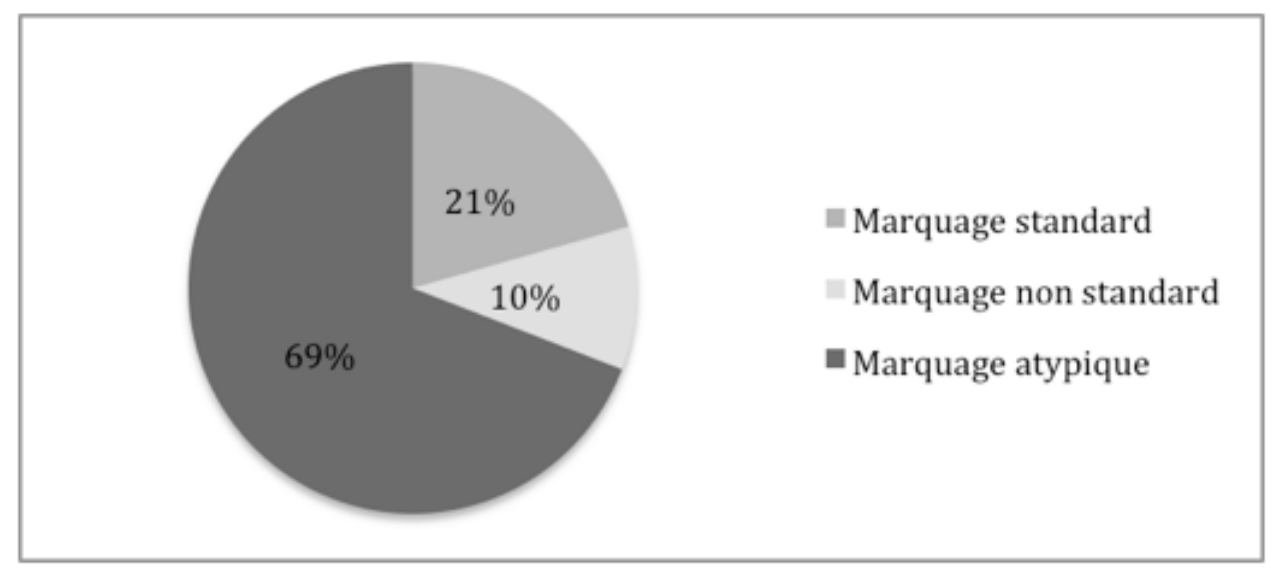

Graphique 2. Syntagmes nominaux pluriels avec des éléments pré- ou post-nucléaires

Pour autant, il ne s'agit pas d'énumérer seulement les possibilités de production de la marque morphologique du pluriel, mais, comme nous l'avons dit précédemment, 
d'observer dans quels contextes elles se produisent, en confrontant l'analyse morphologique à l'analyse phonologique (étant donné que la marque morphologique du pluriel en PB se fait avec une fricative [s] ou [z] au milieu et/ou à la fin des syntagmes nominaux). Ainsi, un phénomène qui, à première vue, semble être motivé par la difficulté chez le jeune enfant de produire la fricative au milieu du syntagme - ce qui pourrait être aussi le cas avec la suppression de la fricative dans dua(s) orelhas (deux oreilles) et doi(s) pés (les deux pieds) - prend une dimension morphosyntaxique avec l'énoncé suivant : «o meninos tam(b)éns correros $»^{9}$ ("les garçons aussi courront») - il se produit une sorte d'hypergénéralisation de l'utilisation du morphème pluriel, selon laquelle des éléments qui ne devraient pas recevoir le $\{-s\}$ (l'adverbe também (aussi) et la forme verbale correram (ont couru) sont également pluralisés, bien que le déterminant reste au singulier.

Ce sont ces énoncés singuliers qui incitent le chercheur à s'interroger sur les autres marques : faut-il comprendre que, dans ce cas, l'enfant a simplement supprimé la fricative dans doi(s) pés ou bien qu'il l'a considérée comme une marque du pluriel et qu'il l'a supprimée comme dans o(s) meninos (le garçons), a bolsas (le sacs) et o olhos (le yeux) ? Ces observations qui ont été faites à partir d'une étude de cas orientent désormais les analyses d'un autre corpus longitudinal monolingue (PB), d'un corpus bilingue (PB/ français) et d'un corpus monolingue français ${ }^{10}$. L'étude qualitative peut donc prendre une dimension quantitative, sans perdre de vue la - riche - singularité des données.

Une autre ressource intéressante du logiciel CLAN est le comptage automatique du MLU ( Mean Length of Utterance), en français, le comptage de la Longueur Moyenne des Énoncés (désormais LME). Cette mesure a été proposée par Brown (1973) pour promouvoir une normalisation dans l'évaluation de la production linguistique de l'enfant. À partir du corpus longitudinal de trois enfants américains, l'auteur a pu identifier l'ordre d'acquisition des 14 premiers morphèmes qu'ils produisaient. Il a proposé alors que la complexité des énoncés prenne en compte non seulement le nombre de mots, mais aussi le nombre de marqueurs grammaticaux. Une proposition qui se justifie avec, par exemple, les deux énoncés suivants: "Daddy eat red apple» et «Daddy eats apples ». Le second énoncé, bien que plus court que le premier, contient deux marqueurs grammaticaux de plus : la troisième personne du singulier pour "eats » et le pluriel pour «apples " (Karmiloff \& Karmiloff-Smith, $2003: 115$ ). Ainsi, la LME permettrait de comparer non pas des enfants du même âge, mais des enfants de même niveau de développement linguistique. On sait en effet qu'il existe beaucoup de variabilité dans l'acquisition du langage et le LME pourrait servir à repérer d'éventuels retards de langage ${ }^{11}$.

51 En fait, jusqu'à ce jour, aucun autre moyen - moins controversé et plus efficace - n'a été découvert pour définir un paramètre qui permettrait de mettre les enfants en parallèle, de comparer leurs productions et de procéder peut-être à certaines généralisations au sujet du processus d'acquisition du langage.

Pour illustrer notre discussion, nous avons choisi de donner des exemples tirés de la production langagière d'un seul enfant, A. Cependant, la totalité de notre recherche porte sur les productions de quatre enfants dont on a comparé les productions en termes de mots par énoncés.

53 Nous n'avons pas utilisé la LME conventionnelle (nombre de morphèmes par énoncé) car il y a une différence importante entre le portugais et le français quand il s'agit de la production du pluriel : en portugais, comme nous l'avons déjà dit, la marque du pluriel à l'oral peut être réalisée sur le déterminant, sur le nom, ou sur chacun des deux éléments. 
En français, la marque du pluriel porte seulement sur le déterminant («le»vs. «les»), la marque « s » n'étant pas prononcée à l'oral (« les chiens »), sauf dans le cas des pluriels irréguliers (« un cheval » vs. « des chevaux »).

Toutefois, la transcription du corpus en français est faite de manière traditionnelle, c'està-dire que le $\{s\}$ du pluriel apparaît dans le nom, tandis qu'en portugais, s'il n'y a pas de production à l'oral, le morphème n'apparaît pas dans la transcription. Ainsi, nous retrouvons des différences entre la mesure de morphèmes dans les énoncés comme : les enfants jouent (le \{s\} dans « enfants » n'est pas produit à l'oral mais il est transcrit dans la forme orthographique), as crianças brincam (marquage standard en portugais, le \{s\} est produit avec le déterminant et le nom à l'oral et on le transcrit dans la forme

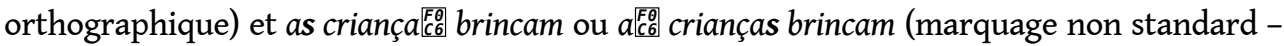
le morphème est produit avec un seul élément du syntagme nominal et transcrit seulement de la façon dont il est produit). Pour cette raison, nous avons décidé de mesurer le nombre de mots par énoncés et non pas le nombre de morphèmes par énoncé pour faire la comparaison entre les deux langues (PB et français) ${ }^{12}$.

\section{Matière à réflexion}

Indépendamment du projet de recherche, seuls certains aspects d'un phénomène donné seront véritablement étudiés, d'autres seront inévitablement exclus, et ce malgré les efforts pour les prendre tous en compte. Autrement dit, il n'est pas approprié de penser qu'il existerait une seule et unique manière de mener une recherche ou qu'il existerait une méthode qui serait la meilleure : la bonne méthode est celle qui répond aux demandes et aux interrogations du chercheur. Le dilemme du chercheur qui s'efforce de choisir entre une recherche qualitative et quantitative n'a pas de sens et opposer ces deux types de recherche se révèle somme toute stérile. En fait, l'important est de se concentrer sur un problème (Günther, 2006).

Comme l'une ou l'autre approche présentent des avantages et des limites, c'est l'adéquation à la question posée par la recherche qui dictera nécessairement le choix. Pour une discipline donnée, la méthode qualitative a comme principal atout le réalisme avec lequel la situation est étudiée, car elle se base sur l'observation. La méthode quantitative et expérimentale garantit pour sa part de plus grandes possibilités de représentativité et de généralisation, étant donné qu'elle traite les données à partir d'échantillons.

57 Ainsi, nous estimons que les deux approches doivent être également valorisées. En d'autres termes, cela signifie que les critères pour évaluer un travail scientifique ne doivent pas se baser seulement sur le choix méthodologique du chercheur, mais également sur la rigueur et la pertinence des réflexions qu'il propose. Les résultats des travaux de chercheurs, comme Piaget, Vygotsky, Clark, Tomasello, parmi beaucoup d'autres, ont montré la pertinence des études qualitatives, ainsi que la rigueur avec laquelle ces données ont été traitées.

Un tel fait montre que la science est en mouvement constant : ce qui est aujourd'hui considéré comme une bonne méthode peut ne plus l'être à l'avenir - et vice versa. Dans le cas de l'approche quantitative, il ne s'agit pas seulement de compter, mais de proposer une analyse pour vérifier la validité d'une hypothèse à partir d'une méthodologie appropriée, dérivée de la méthode expérimentale. 
59 En fait, les deux types d'analyses peuvent s'enrichir mutuellement. En effet, l'analyse qualitative a l'avantage de permettre dans son propre champ d'application, un travail de quantification (ou une analyse statistique descriptive) afin d'éclairer l'hypothèse initiale, alors que l'approche quantitative peut tirer parti de la profondeur des analyses qualitatives. Le chercheur qui associerait ces deux types de recherche aurait en main des généralisations plus probantes, car il tiendrait compte également des singularités. C'est ce que l'on essaie de mettre en œuvre dans les recherches en acquisition du langage en général et c'est ce que l'on a essayé de démontrer de manière illustrative avec l'étude sur le morphème de pluriel chez un enfant brésilien. Ainsi, pour reprendre les questions posées au début de cet article, on peut dire que :

un travail de recherche qui adopte une approche mixte doit proposer des questions qui exigent vraiment les deux types de résultats (qualitatif/quantitatif), sans chercher à valider l'une ou l'autre approche. Dans le cas de la recherche présentée sur l'acquisition $\mathrm{du}$ pluriel, elle est essentiellement qualitative et l'utilisation de l'outil informatique ne fait que donner plus de visibilité à nos résultats qualitatifs. Le quantitatif s'insère donc dans l'approche qualitative.

61 Si l'on posait d'autres questions à partir de ces données - par exemple le phénomène apparaît-il chez un bien plus grand nombre d'enfants, dans un échantillon de 30 enfants? -, on pourrait faire appel à une double approche quantitative et qualitative, chaque approche ayant la même importance. Ainsi, chaque approche n'a pas forcément besoin d'être complétée, sauf, encore une fois, s'il s'agit de répondre à des hypothèses de recherche très spécifiques.

62 Après tout, même si ces deux positions peuvent être potentiellement complémentaires dans le cadre de certains types de recherche, il faut tenir compte du fait que chacune d'elles permet d'aboutir à des résultats valables et pertinents (Valsiner, 2000). En ce qui concerne les données qualitatives, nous n'avons pas besoin de les compléter avec des quantités puisqu'elles peuvent répondre à notre question de recherche, tout en permettant un type de généralisation à partir d'un corpus composé d'enfants « différents » (dans nos exemples, la comparaison d'un enfant français monolingue, d'un enfant brésilien monolingue et d'un enfant franco-brésilien bilingue).

63 L'enjeu de ces réflexions consiste finalement, pour les chercheurs en acquisition du langage à renforcer leurs positions méthodologiques en se munissant d'arguments pour justifier leurs choix, et ce, quelle que soit leur approche ${ }^{13}$.

\section{BIBLIOGRAPHIE}

Bakhtine M., 1986. The Problem of Speech Genres. In Speech Genres and Other Late Essays. Translated by Vern W. McGee, Austin: University of Texas Press. 60-102.

Bauer M. W., 2008. Análise de conteúdo clássica: uma revisão. Em M. W. Bauer \& G. Gaskell (eds.), Pesquisa qualitativa com texto, imagem e som - um manual prático. Petrópolis: Vozes. 189-217. 
Bruner J. S., 1984. Contexts and formats. In M.Moscato, G. Pieraut-Le Bonniec (eds). Le Langage, construction et actualisation.

Bruner J. S., 2004. Comment les enfants apprennent à parler. Paris: Retz.

Brunet É., 2007. Le corpus comme une boule. In F. Rastier et M. Ballabriga (éds), Corpus en lettres et sciences sociales. Des documents numériques à l'interprétation. PUM : Toulouse.

Brown R., 1973. A first language. the early stages. Cambridge: Harvard University Press.

Castro A. \& Ferrari-Neto J., 2007. Um estudo contrastivo do PE e do PB com relação à identificação da informação de número no DP. Letras de Hoje. Vol. 42-1, 65-76.

Del Ré A., à par. Pluralismo teórico-metodológico em Aquisição da Linguagem: o lugar do sujeito e da linguagem nas pesquisas. In A. Gonçalves et M. L. Góis (orgs) Os métodos e os dados nas ciências da linguagem. São Paulo: Mercado de Letras.

Del Ré A., 2012. Abertura: O pluralismo teórico em aquisição de linguagem e suas implicações para a metodologia, dados e ética na pesquisa. In A. Del Ré e M. Romero (orgs) NALingua do outro: estudos interdisciplinares em aquisição de linguagens. São Paulo: Cortez. 15-24.

Duarte T., 2009. A possibilidade da investigação a 3: reflexões sobre triangulação (metodológica). CIES e-Working paper no. 60. Lisboa. Disponível em: http://www.cies.iscte.pt/destaques/documents/ CIES-WP60_Duarte_003.pdf

Ferrari-Neto J., 2003. Reconhecimento do número gramatical e processamento da concordância de número no sintagma determinante na aquisição do português brasileiro. Thèse (Master). Rio de Janeiro : PUC-Rio.

Flick U., 2009a. Desenho da pesquisa qualitativa. Porto Alegre: Artmed.

Flick U., 2009b. Qualidade na pesquisa qualitativa. Porto Alegre: Artmed.

Gesell, 1940. The first five years of life : a guide to the study of the preschool child. London : Methuen.

González Rey F. L. G., 2005. A pesquisa qualitativa em psicologia - caminhos e desafios. São Paulo: Cengage Learning.

Günter, 2006. Pesquisa Qualitativa Versus Pesquisa Quantitativa: Esta é a Questão? Psicologia: Teoria e Pesquisa, V. 22, Mai-Ago, n. 2, 201-210.

Hilário R. N., 2012. A marcação de plural na fala da criança pequena. In A.Del Ré e M. Romero (orgs) Na língua do outro: estudos interdisciplinares em aquisição de linguagens. São Paulo: Cortez. 33-54.

Karmiloff K. \& Karmiloff-Smith A., 2012. Comment les enfants entrent dans le langage. Paris : Retz.

Klee T. \& Fitzgerald M. D., 1985. The relation between grammatical development and mean length of utterance in morphemes. Journal of Child Language 12, 251-69.

Lee K. \& Karmiloff-Smith A., 2002. Macro and microdevelopment research: Assumptions, research strategies, and utilities. Em N. Granott \& J. Parziale (eds.).

MacWhinney B. \& Snow C., 1990. The child language data exchange system: an update, Journal of child language, Vol. 17, 457-472.

Marková I., Linell P., Grossen M. \& Salazar-Orvig A., 2007. Dialogue in focus groups: exploring socially shared knowledge. Londres: Equinox.

Mayaffre D., 2002. L'Herméneutique numérique, L'Astrolabe www.uottawa.ca/academic/ arts/ astrolabe/articles/art0031.htm 
Mazière F. \& Guilhaumou J., 2010. Ainsi nous qui sommes dans le Mississipi. In Paveau M.-A. (éd), La théorie du discours. Fragments d'histoire et de critique, Semen 29, 69-88.

Meline T. J. \& Meline N. C., 1981. Normal variation and prediction of mean length of utterance from chronological age. Perceptual and Motor Skills 53, 376-8.

Parisse C. \& Le Normand M.-T., 1998. Traitement automatique de la morphosyntaxe chez le petit enfant. Glossa 61, 22-9.

Parisse C. \& Le Normand M.-T., 2000. Automatic disambiguation of morphosyntax in spoken language corpora. Behavior Research Methods, Instruments, \& Computers 32, 468-81.

Parker M. D. \& Brorson K., 2005. A comparative study between mean length of utterance in morphemes (MLUm)and mean length of utterance in words (MLUw). First Language 25, 365-376.

Sato et al., 2007. Sampling Reconsidered : Ideographic Science and the Analysis of Personal Life Trajectories. In Valsiner, J. Rosa, A. (eds.) The Cambridge Handbook of Sociocultural Psychology. Cambridge University Press.

Schwandt T. A., 2006. Três posturas epistemológicas para a investigação qualitativa. Em N. E. Denzin \& Y. S. Lincoln (eds.), O planejamento da pesquisa qualitativa: teorias e abordagens (pp. 195-217). Porto Alegre: Artmed.

Valsiner J., 2007. Approaches to culture: semiotic bases for Cultural Psychology. In: J. Valsiner, Culture in minds and societies: foundations of Cultural Psychology (pp. 19-74). Sage: New Delhi.

Valsiner J. \& Rosa A., 2007. The myth and beyond: ontology of psyche and epistemology of psychology. In J. Valsiner \& A. Rosa (eds.), The Cambridge handbook of sociocultural psychology (pp. 23-39). Cambridge, New York: Cambridge University Press.

Vygotsky L. S., 1986. Thought and Language. Trans. Alex Kozulin. Cambridge (Massachusetts): The M.l.T. Press.

Vološinov V. N., 1973. Marxism and the philosophy of language. Trad. Ladislav Matejka and I. R. Titunik. Cambridge/Massachusetts; London/England: Harvard University Press.

Vološinov V.N., 1976. Discourse in life and discourse in art (Concerning sociological poetics). Trans. I. R. Titunik. In: Freudianism: a marxist critique. Ed. I. R. Titunik and Neal H. Bruss. New York: Academic. Rev. Ed. Indianapolis: Indiana UP. 93- 116.

\section{NOTES}

1. Nous ajoutons deux expériences personnelles qui manifestent un tel jugement de valeur. Le refus d'un travail qui avait été soumis à évaluation pour une présentation à un congrès, ainsi que celui d'un article scientifique à l'étranger avec comme seule justification qu'il s'agissait d'une étude qualitative. Nous confirmons également l'importance de cette question pour le thème : Complémentarité des approches quantitatives et qualitatives dans l'analyse des discours ? qui a conduit à cette publication - même si, dans ce cas, l'accent est mis sur l'analyse des discours.

2. L'analyse assistée par ordinateur permet tout aussi bien de fournir des données objectives et quantifiées que de proposer au chercheur des pistes de réflexions, en faisant ressortir des éléments qu'il ne cherchait pas nécessairement (Mayaffre, 2002). Mais l'utilisation de ces programmes ne dispense pas le chercheur des présupposés épistémologiques de sa discipline et de toute considération théorico-méthodologique.

3. Même si les données qualitatives ne permettent pas l'induction au sens strict.

4. Disponible à l'adresse suivante : http://childes.psy.cmu.edu 
5. Le groupe GEALin (Groupe d'Études en Acquisition du Langage) de la Faculté des Sciences et Lettres de l'Université de l'État de São Paulo, en Araraquara (FCLAr-UNESP), fait partie du groupe NALíngua et compte sur la participation de 14 étudiants dont les projets s'inscrivent dans la ligne de recherche: "Identité et altérité dans le langage de l'enfant ». L'objectif du groupe est de discuter de la question de la place que prend l'enfant à l'égard de l'autre dans son discours et ce à partir de données recueillies longitudinalement et transversalement.

6. Le groupe de recherche NALingua-CNPq (Centre d'Études en Acquisition du Langage) existe depuis 2008 et compte actuellement 12 enseignants-chercheurs et 18 étudiants. Le groupe a comme thème de discussion, le rapport entre l'identité et l'altérité qui marque l'entrée de l'enfant dans la langue, ainsi que les processus qui marquent un changement de placement du locuteur dans le discours (oral/écrit) de l'enfant. Il réunit des chercheurs de diverses disciplines (linguistique, psychologie, phono-audiologie, éducation) avec des approches théoriques différentes et complémentaires (l'approche cognitiviste, lacanienne, générative, énonciative et dialogique). Ces chercheurs se proposent d'apporter leurs contributions en analysant le même corpus : depuis 2008, les données recueillies régulièrement auprès de six enfants âgés de 0 à 7 ans, dans des conditions naturelles et dans un contexte familial et scolaire.

7. Pour plus d'information, consulter respectivement les sites http://colaje.scicog.fr/ et http:// www.univ-paris3.fr/anr-diaref-37421.kjsp

8. En portugais du Brésil le morphème du pluriel peut être ajouté à tous les éléments du syntagme nominal - marquage standard, comme dans as crianças (les enfants) - ou seulement au premier élément du syntagme - marquage non standard, comme dans as criança因 (les enfants). Il est réalisé comme une fricative [s] quand il précède une consonne et comme une fricative $[\mathrm{z}]$ quand il précède une voyelle.

9. Énoncé produit par A. (2;7.03).

10. Recherche de doctorat en cours.

11. Cette proposition lancée dans les années soixante-dix a d'abord été bien reçue (voir les références citées par Parker \& Brorson, 2005), avant d'être critiquée par la suite (Meline \& Meline, 1981 ; Klee et Fitzgerald, 1985 entre autres). Aujourd'hui, elle est de nouveau adoptée en Europe et aux États-Unis et est même considérée comme un préalable à toute publication dans des revues internationales.

12. Ces résultats ne seront pas montrés dans cet article dont ce n'est pas le but. Cependant, nous pensons que dans une approche quantitative, il faut discuter la question de la méthode de comparaison entre les langues.

13. Nous tenons à remercier le Professeur Frédéric François et Christelle Dodane pour les réflexions auxquelles leurs précieux conseils nous ont conduites et, surtout, pour le regard critique qu'ils ont porté sur cet article. Nous remercions également les groupes NALingua (CNPq), GEALin/ UNESP, COLAJE et DIAREF pour les discussions et leurs collaborations.

\section{RÉSUMÉS}

Quelle est la place des données qualitatives ? Comment doit-on envisager une approche mixte? Faut-il compléter l'approche quantitative par une approche qualitative (Mazière et al., 2010) ou faire le contraire? Comment ces deux approches peuvent-elles être combinées dans le cadre d'une même recherche en acquisition du langage? Quelles sont les possibilités de généralisation 
qu'elles permettent? Nous illustrerons ces discussions théoriques par une recherche essentiellement qualitative sur l'acquisition des morphèmes du pluriel en portugais du Brésil (PB) chez un jeune enfant entre 1 an 11 mois et 2 ans 7 mois (Hilário, 2012), dont les productions linguistiques sont intrinsèquement liées à leur caractère dialogique (Bakhtine \& Vološinov, 1973) et à l'interaction avec les interlocuteurs (Bruner, 2004 ; Vygotsky, 1986). Nous montrerons que l'utilisation d'un outil informatique (CLAN) sert à donner plus de visibilité à nos résultats quantitatifs même s'ils s'insèrent dans l'approche qualitative. Nous verrons enfin qu'il est possible d'arriver à un type de généralisation (statistique ou analytique), même s'il faut tenir compte du fait que chacune d'elles permet d'aboutir à des résultats valables et pertinents (Valsiner, 2000).

What is the role of qualitative data? How should mixed approaches be considered? Is it necessary to complement the quantitative approach by the qualitative approach (Maziére et al., 2010) or the opposite? How can these two approaches be combined in a Language Acquisition research? What are the possibilities of generalization that these approaches provide? Besides those necessary theoretical discussions, our starting point is a qualitative research on the acquisition of the plural morpheme in Brazilian Portuguese (BP) in children between 1 year 11 months old and 2 years 7 months old (Hilário, 2012), whose linguistic production is intrinsically connected to their dialogic aspect (Bakhtin \& Vološinov, 1973) and to the interaction among interlocutors (Bruner, 2004; Vygotsky, 1986). In this research through the usage of a computational tool (CLAN) it is expected to have more visibility of the quantitative results even if they are in a qualitative approach. Although, it should be taken into account the fact that each approach can achieve valid and relevant results (Valsiner 2000), i.e., with any given method it is possible to reach a kind of generalization (statistical or analytical).

INDEX

Mots-clés : Acquisition du langage, Dialogisme, Approche Mixte, Généralisation, Pluriel

Keywords : Language Acquisition, Dialogism, Mixed Approach, Generalization, Plural

\section{AUTEURS}

ALESSANDRA DEL RÉ

UNESP (Brésil)

ROSÂNGELA NOGARINI HILÁRIO

UNESP/FAPESP (Brésil) 